\title{
Analysis on Relief Efficiency and Influencing Factors of Large-scale Geological Disasters
}

\author{
La Ruifang ${ }^{1, a^{*}}$, Zhang Zaixu ${ }^{1, b}$, Bai Pengfei ${ }^{2, c}$ \\ ${ }^{1}$ School of Economics and Management, China University of Petroleum, Qingdao Shandong 266580, China \\ ${ }^{2}$ School of Management And Engineering, Capital University of Economics and Business, Beijing 100070, China \\ a*laruifang@163.com \\ ${ }^{b}$ zzx1766@126.com \\ cbpfnet@126.com
}

\begin{abstract}
The sudden complexity and destruction of major geological disasters determine the necessity of improving disaster relief efficiency. However, due to the relative lack of geological disaster relief data, the research on the relief efficiency evaluation of major geological disasters is insufficient. Based on the data of 18 rescue operations of major geological disasters in China from 2015 to 2019, this paper measure the disaster relief efficiency and analyze the influencing factors by using the super-efficiency SBM model and Tobit regression model with non-expected output. The research suggests several results: the average efficiency of rescue operations for 18 major geological disasters was 0.671 , and that of disasters below the average efficiency was $67 \%$; The average efficiency of several geological disaster relief operations in 2019 was 0.73 , which explaining that after the establishment of the ministry of emergency management, the efficiency of geological disaster relief in China has been improved obviously; However, the long rescue period, redundant rescue personnel and unreasonable allocation of rescue equipment are the reasons why the rescue efficiency is relatively low for heavy and severe geological disasters. The research also found that the number of disaster victims and people trapped, the per capita GDP, the total population of the county, the county area and the highway density have a significant positive relationship with the rescue efficiency, whereas the disaster time, population density and highway mileage are negatively correlated with the rescue efficiency. In view of the above problems, policy suggestions are put forward to further improve the relief efficiency of China's major geological disasters.
\end{abstract}

Keywords: Large-scale geological disasters, Rescue efficiency, Influencing factors, DEA-SBM, Tobit.

\section{重特大地质灾害救援效能及其影响因素分析}

\author{
喇芯芳 $1, \mathrm{a}^{*}$ 张在旭 ${ }^{1, b}$ 白鹏飞 $2, \mathrm{c}$ \\ 1 中国石油大学 (华东) 经济管理学院, 山东 青岛 266580 \\ 2 首都经济贸易大学 管理工程学院, 北京 100070 \\ a*laruifang@163.com \\ bzzx1766@126.com \\ cbpfnet@126.com
}

摘要

重特大地质灾害的突发性、复杂性、毁灭性决定了提高灾难救援效能的必要性，但由于地质灾害救援数据相对 缺乏，专门针对重特大地质灾害救援效率评估的研究存在不足。本文基于 2015-2019 年我国发生的 18 起重 特大地质灾害救援行动数据, 运用包含非期望产出的超效率 SBM 模型和 Tobit 回归模型对灾害救援效能进行 了测度并分析其影响因素。研究结果表明：18 起重特大地质灾害救援行动的平均效率为 0.671 , 低于平均效率 值的灾害占样本总数 $67 \%$; 2019 年的几次地质灾害救援行动平均效率为 0.73 , 说明应急管理部成立后我国地 
质灾害救援效率提升明显; 而救援周期过长、救援人数穴余、救援装备分配不合理是重特大地质灾害救援效率 相对较低的原因。研究还发现, 受灾人数、被困人数、人均 GDP、县城总人口、县城面积、公路密度与救援效 率有显著的正向关系，而灾害发生时间、人口密度、公路里程数与救援效率呈负相关。针对上述问题提出进一 步提升我国重特大地质灾害救援效能的政策建议。

关键词: 重特大地质灾害; 救援效能; 影响因素; DEA-SBM; Tobit

\section{1.前言}

重特大地质灾害的发生, 往往具有较强的隐蔽性、 突发性和较大的破坏性, 并且多发生在恶劣环境下, 给灾后救援工作带来极大困难 ${ }^{[1]}$ 。存在救援效率不高、 投入资源攵余浪费、救援协同迟滞等问题 ${ }^{[2]}$ 。

开展重特大地质灾害救援效率评估工作是我国 自然灾害频发基本国情的需要, 然而目前学术界对地 质灾害救援效率的研究很少, 重特大地质灾害救援评 估工作尚处在起步阶段。Abidi H（2014）和 Blecken A（2010）等人指出目前对于人道主义救援效率的评 价十分缺乏 ${ }^{[3-4]}$, Schulz S（2009）构建了灾后物流运 作效率的评价指标 ${ }^{[5]}$, Üstün A（2015）和 Acimovic J （2016）提出 DEA 方法可评估人道主义组织救灾效 率 ${ }^{[6-7]}$ 。目前, 相对于国外应急领域的研究, 我国在该 领域的研究起步较晚, 但已有相关专家学者对其进行 了探索并取得了一定进展, 研究成果主要包括三个方 面: 一是针对某一起灾害事件救援的过程、方式及救 援各方扮演的角色等进行相关定性分析 ${ }^{[8]}$; 二是从人 道主义救援供应链的角度测算灾害救援效率 ${ }^{[9-10]}$; 三 是从资源配置角度评价救援工作效率 ${ }^{[11]}$ 。

对于影响救援效率因素的研究聚焦于分析单起 自然灾害下某一人道主义组织的救援效率影响因素,

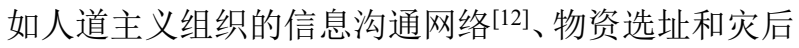
救援资金 ${ }^{[13]}$ 以及受灾国的地理位置 ${ }^{[14]}$ 。

目前关于地质灾害大多数研究聚焦于滑坡、泥石 流等过程实录 ${ }^{[15]}$ 、成因机制[16]、风险与损失评估 ${ }^{[17]}$ 以 及灾害预测 $[18]$ 等方面。因此需要从定量、客观、宏观 的角度衡量我国重特大地质灾害救援效率及其影响 因素, 以期提高灾害救助工作的科学性、针对性和时 效性。

\section{2. 基于 SBM 模型的救援效能分析}

\section{1. 指标与模型构建}

效率评价方法一般分为两种, 即参数前沿方法和 非参数前沿方法, 数据包络分析 (DEA) 是一种客观、 成熟、应用广泛的非参数前沿方法 ${ }^{[19-20]}$, 不需要考虑 随机误差问题, 可以满足灾害救援效率评估的客观约 束。
本研究将采用包含非期望产出的超效率 SBMDEA 模型对重特大地质灾害救援效率进行评估, 使 评估结果更加准确, 具有可信度。SBM 模型如下:

$$
\begin{gathered}
\rho=\min \frac{1-\frac{1}{m} \sum_{i=1}^{m} \frac{s_{i}^{-}}{x_{i o}}}{1+\frac{1}{q_{1}+q_{2}}\left(\sum_{r=1}^{q_{1}} \frac{s_{r}^{+}}{y_{r o}}+\sum_{t=1}^{q_{2}} \frac{s_{t}^{b-}}{b_{r o}}\right)} \\
\text { s.t. }\left\{\begin{array}{l}
x_{o}=X \lambda+S^{-} \\
y_{o}=Y \lambda-S^{+} \\
b_{o}=B \lambda+S^{b-} \\
\lambda \geq 0, S^{-} \geq 0, S^{+} \geq 0
\end{array}\right.
\end{gathered}
$$

其中: $\rho$ 为效率值; ${ }^{i o}$ 为输入值; ${ }^{r o}$ 为期望产出; $q_{1} 、 q_{2} 、 m$ 分别表示期望产出、非期望产出、投入指 标的个数; $S^{-} 、 S^{+} 、 S^{b-}$ 分别表示投入㫕余量、期望 产出不足量和非期望产出超标量; $\lambda$ 为权重向量; $X$ 、 $Y 、 B$ 分别表示投入指标、期望产出指标、非期望产 出指标构成的向量。 $0 \leq \rho \leq 1$, 当且仅当 $\rho=1$ 时决策 单元有效, 否则为非有效单元。

基于非期望产出 SBM 模型评价方法最关键的一 步是评价指标的选取, 本研究在指标选取过程中考虑 到指标的全面性、代表性以及科学可行性, 以减少受 灾地区人员伤亡和满足受灾地区人员避险需求为侧 重点, 初步选取人力、财力、物力、时间方面的 8 个 指标作为救援投入指标, 以遇难、获救人数以及土石 方作业、抢险工程量等方面的 7 个指标为救援产出指 标。

在此基础上, 本文采用群组决策特征根法 (GEM), 邀请地质灾害救援领域 13 位专家学者对指标体系打 分, 用 MATLAB 计算得出最大特征根为单根, 进行 关键指标的甄选, 剔除重要性小于 0.3000 的指标, 并 考虑到指标需要反映出救援效能的差异, 指标所需数 据尽可能客观完整且结构化程度高, 最后经过篮选, 本研究选取救援人数、救援时间和大型救灾机械这 3 个反映救灾规模及救灾难度和复杂程度的指标, 作为 衡量救援投入资源的量化指标, 而将紧急转移安置人 数、死亡与失联人数这 2 个能够综合反映地质灾害严 重程度、救援难度及迫切性的指标作为衡量救援产出 
的量化指标。其中, 紧急转移安置人数为期望产出, 死亡失联人数为非期望产出。

在相同灾害严重程度下, 完成救援任务所需投入 数量越小, 则救援效能越高。

$$
h_{\text {效能 }}=\frac{u_{1} \text {. 救援人数 }+u_{2} \cdot \text { 救援时间 }+u_{3} \text { ・救援装备 }}{v_{1} \text {. 灾情指标 } 1+v_{2} \text {. 灾情指标 } 2}
$$

综上分析，重特大地质灾害救援效能评估指标体 系如表 1 。

表 1 重特大地质灾害救援效能评估指标体系

\begin{tabular}{|c|c|c|c|c|}
\hline 类型 & 编号 & 名称 & 单位 & 说明 \\
\hline \multirow{3}{*}{$\begin{array}{c}\text { 救援指标 } \\
\text { Input }\end{array}$} & 11 & 救援总人数 & 人 & 抢险救灾力量, 包括消防、武警、军队 \\
\hline & 12 & 救援天数 & 天 & $\begin{array}{l}\text { 从灾害发生到官方宣告救援行动结束所持续的时间, 救援难度越 } \\
\text { 大, 搜救范围越广, 救援现场越复杂, 则救援时间持续越长 }\end{array}$ \\
\hline & 13 & 大型救灾机械 & 台/套 & 土石方作业中挖掘机、推土机、铲车、装载机等装备数目 \\
\hline 灾情指标 & 01 & 转移安置人数 & 人 & 反应灾害的破坏威力与规模 \\
\hline Output & OBad & 死亡失联人数 & 人 & 最直接的反应灾害严重程度, 人数越多, 灾害的严重程度越大 \\
\hline
\end{tabular}

\section{2. 数据来源与处理}

为客观评估救援行动效能, 本文采集了 20042019 年中国发生的 87 起滑坡、泥石流事件救援行动 数据, 以受灾面积大, 波及范围广, 受灾人口多, 涉 及的救援任务繁多, 救援难度大, 造成了巨大的经济 损失为原则, 笁选了近五年发生的 18 个极具代表性 的地质灾害救援行动案例。指标数据主要来自 20152019 年度全国重大地质灾害事件与应急避险典型案 例[21]、中华人民共和国应急管理部、自然资源部、民 政部等官网灾情简报; 各省统计年鉴; 各省、市、县 级政府官网、地方报纸、广播电台及相关新闻网站等。

收集数据过程中, 由于重特大地质灾害救援效率 各评价指标的性质不同, 具有不同的量纲和数量级,
为了保证结果的可靠性, 本研究采用对数 Logistic 模 式对原始数据进行标准化处理, 处理方法如下:

$$
\text { 新数据 }=\ln (\text { 原数据 })
$$

\section{3. 效能测度结果}

利用 DEA-SOLVER Pro5.0 软件测算这 18 起重 特大地质灾害救援行动的效能, 结果如表 2 所示。综 合来看, 18 起重特大地质灾害救援行动的平均效率为 0.671 , 样本中低于平均效率值的地质灾害有 12 起, 即样本中救援效率处于平均效率值以下的地质灾害 占样本总数 $67 \%$, 说明我国重特大地质灾害救援效率 一般, 救援效率亟待提升。

\begin{tabular}{|c|c|c|c|c|c|}
\hline 序号 & 时间 & 救援行动 & 简称 & 效能值 & 排序 \\
\hline 1 & 2019 & 四川汶川 820 泥石流 & $2019 w c 0820$ & 1.003 & 3 \\
\hline 2 & 2019 & 浙江永嘉 810 堰塞湖 & 2019zj0810 & 0.719 & 5 \\
\hline 3 & 2019 & 四川成昆铁路 814 泥石流 & $2019 s c 0814$ & 0.682 & 6 \\
\hline 4 & 2019 & 贵州水城 723 滑坡 & 2019gz0723 & 0.602 & 11 \\
\hline 5 & 2019 & 山西乡宁 315 滑坡 & $2019 s \times 0315$ & 0.646 & 8 \\
\hline 6 & 2018 & 金沙江 113 滑坡 & 2018jsj1103 & 0.655 & 7 \\
\hline 7 & 2018 & 雅鲁藏布江 1017 堰塞湖 & 2018ylzbj1017 & 1.008 & 2 \\
\hline 8 & 2018 & 金沙江 1011 白格堰塞湖 & 2018jsj1011 & 0.783 & 4 \\
\hline 9 & 2018 & 云南麻栗坡 92 泥石流 & 2018yn0902 & 0.482 & 16 \\
\hline 10 & 2018 & 湖南阮陵 630 滑坡 & $2018 \mathrm{hn} 0630$ & 0.512 & 15 \\
\hline 11 & 2017 & 青海玛沁 830 滑坡 & 2017qh0830 & 0.608 & 9 \\
\hline 12 & 2017 & 贵州纳雍 828 滑坡 & $2017 g z 0828$ & 0.602 & 10 \\
\hline 13 & 2017 & 甘肃文县 87 泥石流 & $2017 g s 0807$ & 1.302 & 1 \\
\hline 14 & 2017 & 四川茂县 624 滑坡 & $2017 \operatorname{sc} 0624$ & 0.527 & 13 \\
\hline 15 & 2016 & 浙江丽水 928 滑坡 & 2016zj0928 & 0.514 & 14 \\
\hline 16 & 2016 & 福建三明 58 泥石流 & 2016fj0508 & 0.575 & 12 \\
\hline 17 & 2015 & 陕西山阳 812 山体滑坡 & $2015 s \times 0812$ & 0.446 & 17 \\
\hline 18 & 2015 & 浙江丽水 1113 山体滑坡 & 2015zj1113 & 0.413 & 18 \\
\hline
\end{tabular}

\section{表 2 2015-2019 年重特大地质灾害救援效能}


而中国的灾害救援 “把抢救生命作为首要任务”, 一旦发生地质灾害，无论规模大小，均举全城之力进 行救援，但是从救援整体情况来看，虽然政府投入大 量的人力、财力、物力，但是仍旧不能满足重大地质 灾害应急救援的需要。救援周期过长、救援人数圥余、 救援装备分配不合理导致的投入元余和期望产出不 足是是我国重特大地质灾害救援效率相对较低的原 因。

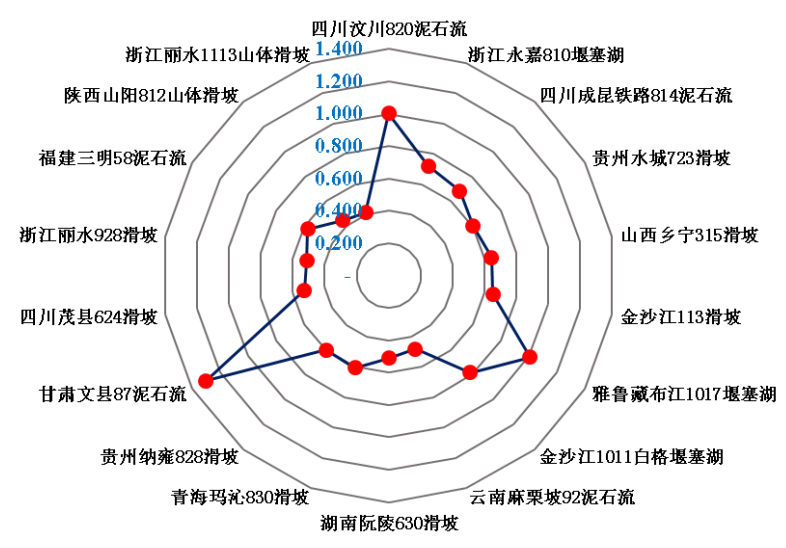

图 118 次救援行动效能值

从图 1 可以看出, 18 起重特大地质灾害救援效 率波动较大。可能有以下原因: (1) 灾难的严重程度、 灾难的类型影响到救援效率; (2)不同地区发生的地质 灾害, 由于地理环境等因素会影响到救援效率。

\section{3. 基于 Tobit 模型的效能影响因素分析}

\section{1. 指标与模型构建}

由于灾害救援效率值不是连续性数值, 而 Tobit 模型符合截尾回归模型的特点 [22], 当前关于灾害救援 效率评价及影响因素的研究主要采用 DEA-Tobit两阶 段模型, 第一阶段用 DEA 求解 DMU 的效率值, 第 二阶段用 Tobit 回归分析效率值的影响因素。将 SBM 模型测算的效率值 Y 作为被解释变量, 结合影响因素 建立回归方程：

$$
\begin{gathered}
\mathrm{y}_{i}=\beta_{o}+\beta_{1} x_{i 1}+\beta_{2} x_{i 2}+\ldots+\beta_{\mathrm{k}} x_{i k}+\mu_{i} \\
Y_{i}=y_{i}, \text { if } y_{i} \in(0,1] \\
Y_{i}=0, \text { if } y_{i} \in(-\infty, 0) \\
Y_{i}=1, \text { if } y_{i} \in(1,+\infty)
\end{gathered}
$$

其中: ${ }_{i}$ 为被解释变量 (重特大地质灾害救援效 率); $\beta$ 是回归系数; $x_{i}$ 为解释变量; $\mu_{i}$ 为随机误差 项。一般情况下, 回归系数为正, 则影响是积极的, 如果为负则是消极的。

结合重特大地质灾害发生的特点, 考虑到指标选 取原则以及数据的可获取性, 本文从灾害规模、经济 发展水平、人口状况、交通运输水平四个方面提出 10 种可能的影响因素指标, 为使影响因素的析取更加精 确, 指标细化到灾害发生地县级相关数据, 统计数据 来源于《中国统计年鉴》、《国民经济和社会发展统计

\begin{tabular}{|c|c|c|c|c|c|}
\hline 变量 & 影响因素 & 指标 & 说明 & $\begin{array}{l}\text { 编 } \\
\text { 号 }\end{array}$ & 单位 \\
\hline 被解释变量 & & 效率值 & 地质灾害救援效率值 & Y & - \\
\hline \multirow{10}{*}{ 解释变量 } & \multirow{3}{*}{ 灾害规模 } & $\begin{array}{c}\text { 灾害发生 } \\
\text { 时间 }\end{array}$ & $\begin{array}{l}7: 00-16: 00 \text { 为 } 1 ; 16: 00-20: 00 \text { 为 } 2 ; 20: 00- \\
23: 00 \text { 为 } 4 ; 23: 00-5: 00 \text { 为 } 5 ; 5: 00-8: 00 \text { 为 } 3\end{array}$ & $\mathrm{X}_{1}$ & - \\
\hline & & 受灾人数 & 受灾已转移安置人数 & $\mathrm{X}_{2}$ & 人 \\
\hline & & 被困人数 & 被困获救人数 & $X_{3}$ & 人 \\
\hline & 经济发展水平 & 人均 GDP & 灾害发生地县级人均 GDP & $\mathrm{X}_{4}$ & 元 \\
\hline & \multirow{4}{*}{ 人口状况 } & 人口密度 & 县城总人口/面积 & $X_{5}$ & 人 $/ \mathrm{km}^{2}$ \\
\hline & & 县城总人口 & 县城总人口 & $X_{6}$ & 人 \\
\hline & & 县城面积 & 县城面积 & $\mathrm{X}_{7}$ & $\mathrm{~km}^{2}$ \\
\hline & & 公路里程数 & 受灾地区县城公路里程数 & $\mathrm{X}_{8}$ & km \\
\hline & \multirow[t]{2}{*}{ 交通运输水平 } & 公路密度 & 每百平方公里所拥有的公路总里程数 & $X_{9}$ & $\mathrm{~km} / 100 \mathrm{~km}^{2}$ \\
\hline & & 公路密度 & 每万人所拥有的公路总里程数 & $X_{10}$ & km/万人 \\
\hline
\end{tabular}
公报》、《政府工作报告》等，影响因素指标体系如表 3 所示。

\section{表 3 重特大地质灾害救援效率影响因素指标体系}

\section{2 结果分析}

本文使用 Eviews 8.0 软件对模型进行 Tobit 估计, 实证分析结果如表 4 所示。
从表 4 的模型估计结果来看: 模型的 $\mathrm{R}^{2}$ 值为 0.959 , 意味着 $\mathrm{X}_{1}-\mathrm{X}_{10}$ 可以解释 $\mathrm{Y}$ 的 $95.9 \%$ 变化原因, 对模型进行 $\mathrm{F}$ 检验时发现模型通过 $\mathrm{F}$ 检验 $(\mathrm{F}=16.454$, $\mathrm{p}=0.001<0.05)$, 也即说明模型构建有意义。 
表 4 Tobit 回归分析结果

\begin{tabular}{|c|c|c|c|c|c|c|c|}
\hline 变量 & 相关系数 & Std. Error. & $\begin{array}{c}\text { z- } \\
\text { statistic }\end{array}$ & $\mathbf{P}$ & $R^{2}$ & $A R^{2}$ & $F$ \\
\hline$C$ & 0.208082 & 0.068601 & 3.03321 & $0.0024^{* \star *}$ & \multirow{11}{*}{0.959} & & \multirow{11}{*}{$\begin{array}{c}F(10,7)=16.454 \\
p=0.001\end{array}$} \\
\hline $\mathrm{X} 1$ & -0.038951 & 0.00893 & -4.361984 & $0.0000^{* * *}$ & & & \\
\hline $\mathrm{X} 2$ & $1.15 \mathrm{E}-06$ & 4.81E-07 & 2.401376 & $0.0163^{* *}$ & & & \\
\hline $\mathrm{X} 3$ & 0.000172 & $6.60 \mathrm{E}-05$ & 2.608054 & $0.0091^{* * *}$ & & & \\
\hline$X 4$ & 4.16E-06 & 8.76E-07 & 4.755069 & $0.0000^{\star * *}$ & & & \\
\hline$x 5$ & -0.00204 & 0.000503 & -4.05266 & $0.0001^{* * *}$ & & 0.901 & \\
\hline$x 6$ & 1.14E-06 & 2.13E-07 & 5.331952 & $0.0000^{* * *}$ & & & \\
\hline $\mathrm{X} 7$ & 1.38E-05 & $3.60 \mathrm{E}-06$ & 3.817685 & $0.0001^{* * *}$ & & & \\
\hline$x 8$ & -0.000112 & 2.72E-05 & -4.111046 & $0.0000^{* * *}$ & & & \\
\hline$\times 9$ & 0.002407 & 0.000824 & 2.920864 & $0.0035^{\star * *}$ & & & \\
\hline $\mathrm{X} 10$ & 0.001955 & 0.000218 & 8.961415 & $0.0000^{* * *}$ & & & \\
\hline
\end{tabular}

(1) 在灾害规模方面, 灾害发生时间呈现出 0.01 水平显著性 $(\mathrm{p}=0.0000<0.01)$, 意味着灾害发生时间会 对效率产生显著的负向影响关系, 若发生在白天，救 援响应及时, 救援效率较高, 若发生在凌晨, 睡熟的 人们对于环境警惕性不强, 再加上夜里救援难度大, 救援效率较低; 受灾人数呈现出 0.01 水平显著性 $(p=0.0163<0.05)$, 意味着受灾人数会对效率产生显著 的正向影响关系, 如果假定其他情况不变, 受灾已转 移安置人数每增加 $1 \%$, 救援效率值将增加 0.038951 个百分点; 被困人数呈现出 0.05 水平显著性 $(\mathrm{p}=0.0091<0.01)$, 意味着被困人数会对效率产生显著 的正向影响关系, 被困获救人数每增加 $1 \%$, 救援效 率值将增加 1.12 个百分点。

(2)在经济发展水平方面, 人均 GDP 呈现出 0.01 水平显著性 $(\mathrm{p}=0.0000<0.01)$, 意味着经济发展水平会 对效率产生显著的正向影响关系, 人均 GDP 每增加 $1 \%$, 救援效率值将增加 4.16 个百分点。

（3）在人口状况方面, 人口密度呈现出 0.01 水 平显著性 $(\mathrm{p}=0.0001<0.01)$, 意味着人口密度会对效率 产生显著的负向影响关系, 人口密度越高, 灾害发生 瞬间被埋压人员数越多, 人口密度每增加 $1 \%$, 救援 效率值将降低 0.00204 个百分点; 县城总人口呈现出 0.01 水平显著性 $(\mathrm{p}=0.0000<0.01)$, 意味着县城总人口 会对效率产生显著的正向影响关系, 人口越多, 可发 动的救援力量越多, 县城总人口每增加 $1 \%$, 救援效 率值将增加 1.14 个百分点; 县城面积呈现出 0.01 水 平显著性 $(\mathrm{p}=0.0001<0.01)$, 意味着县城面积会对效率 产生显著的正向影响关系, 县城面积每增加 $1 \%$, 救 援效率值将增加 1.38 个百分点;

(4) 在交通运输水平方面, 公路里程数呈现出 0.01 水平显著性 $(\mathrm{p}=0.0000<0.01)$, 意味着公路里程数 会对效率产生显著的负向影响关系, 公路里程数每增 加 $1 \%$, 救援效率值将降低 0.000112 个百分点; 公路 密度呈现出 0.01 水平显著性 $(\mathrm{p}=0.0001<0.01)$, 意味着
公路密度会对效率产生显著的正向影响关系, 公路密 度每增加 $1 \%$, 救援效率值将增加 0.002 个百分点。

综上所述, 受灾地区的受灾人数、被困人数、人 均 GDP、县城总人口、县城面积、公路密度会对救援 效率值产生显著的正向影响关系，而灾害发生时间、 人口密度、公路里程数会对救援效率值产生显著的负 向影响关系, 我们可以从宏观层面提高重特大地质灾 害救援效率。

\section{4. 结论}

本文运用包含非期望产出的超效率 SBM 模型对 我国 2015-2019 年发生的 18 起重特大地质灾害救援 效率进行了测算, 并对影响效率值的因素进行了探讨, 研究结论如下:

（1）救援效率的评估表明，总体而言，18 起重 特大地质灾害救援行动的平均效率为 0.654 , 低于平 均效率值的灾害占 $61.1 \%$, 说明我国重特大地质灾害 救援效率亟待提升。

(2) 影响因素的实证分析表明, 灾害规模、经济 发展水平、人口状况、交通运输水平对救援效率值都 会产生显著影响, 其中, 受灾人数、被困人数、人均 GDP、县城总人口、县城面积、公路密度会对救援效 率值产生显著的正向影响关系，而灾害发生时间、人 口密度、公路里程数会对救援效率值产生显著的负向 影响关系。

\section{REFERENCES}

[1] Wang, X., Zhou, H. (2018). Progress and Prospect of Statistics and Assessment of Large-scale Natural Disaster Damage and Losses. Advances in Earth Science(09):914-921.

[2] Fang, L., Xia, Y., Yang ,Y., et al. (2016).The Literature Review and Future Prospects of Relief Supply Chain for Unexpected Natural Disasters. Management 
Review, 28(08):238-249.

[3] Klumpp, M., Abidi, H., Leeuw, S. (2014). Humanitarian supply chain performance management: a systematic literature review. Supply Chain Management: An International Journal.

[4] Blecken, A. (2010). Supply chain process modelling for humanitarian organizations. International Journal of Physical Distribution \& Logistics Management, 40(8-9): 675-692.

[5] Schulz, S. F., \& Heigh, I. (2009). Logistics performance management in action within a humanitarian organization. Management Research News, 32(11), 1038-1049.

[6] AK Üstün, \& G Barbarosoğlu. (2015). Performance evaluation of turkish disaster relief management system in 1999 earthquakes using data envelopment analysis. Natural Hazards, 75(2), 1977-1996.

[7] Acimovic, J, Goentzel, A. (2016). Models and metrics to assess humanitarian response capacity. Journal of operations management, 45(7.), 11-29.

[8] Lin, W., Wang, N. (2019).A comparison study on the rescue efficiency in the international waters: a case of the specific waters in the East China Sea. Marine Science Bulletin, 38(04):438-446.

[9] Guan, G. (2016). Research on the Collaboration Mechanism of Humanitarian relief Supply Chain.Doctoral dissertation, Chang'an University, Xi'an, China

[10] Leng, Q. (2018). The Evaluation and Analysis of Natural Disaster Relief Efficiency of International Humanitarian Organizations. Doctoral dissertation, University of Science and Technology of China.

[11] Sun, H., Zhao, J., Liu, T., et al. (2019). Research on the Efficiency Evaluation of Emergency Medical Rescue Process after Earthquake. Chinese Journal of Management Science, 27(01):205-216.

[12] Meesters, K., Walle, B. (2014). Increasing Efficiency of Humanitarian Organizations with Volunteer Driven Information Products. Hawaii International Conference on System Sciences (HICSS). IEEE. 149-158.

[13] Balcik, B., Beamon, B. (2008). Facility location in humanitarian relief. International Journal of Logistics Research \& Applications, 11(2), 101-121.

[14] Wei, J., Zhao, D., Marinova, D. (2013). Disaster Relief Drivers: China and the US in Comparative Perspective. China an International Journal, 11(2):93-116.

[15] Deng, Z., Huang, S., Zhu, G. (2019). Temporal and spatial distribution characteristics analysis for fujian landslide disasters based on disaster data in recent five years. Journal of Geological Hazards and Environment Preservation, 30(04):17-23.

[16] Zhou, J., Zhao, F., Li, H. (2019). Correlational Research between Geological Hazards and impact Factors in Shaanxi Province. Journal of Catastrophology, 2019, 34(02):228-234.

[17] Hong, Z., Li, Y., Zhang, L., et al. (2020). A Method of Regional Geological Hazard Assessment Based on Principle Component Analysis. Journal of Catastrophology, 35(01):118-124.

[18] Niu, H.(2020).Prediction of Landslide Geological Hazards in Soil-rock Contact Zone in Southern Shaanxi. Journal of Catastrophology, 35(01):125130 .

[19] Cooka, W., D. (2009) Data envelopment analysis (DEA) - Thirty years on. European Journal of Operational Research, 192(1):1-17.

[20] Golany, B., Roll, Y. (1989).An application procedure for DEA. Omega, 17(3):237-250.

[21] Geological Disaster Technical Guidance Centre of the Ministry of Natural Resources (2020). 20152019 National Major Geological Disaster Events and Emergency Avoidance Typical Cases. Beijing: Geological Publishing House.

[22] Liu, Y., Wei, J., Xu, J., et al. (2018). Evaluation of the moderate earthquake resilience of counties in China based on a three-stage DEA model. Natural Hazards, 91(2): 587-609. 